\title{
Barrett's Esophagus and Intestinal Metaplasia of Gastric Cardia: Prevalence, Clinical, Endoscopic and Histological Features
}

\author{
Ofelia Daniela Pascarenco ${ }^{1}$, Alina Boeriu ${ }^{1}$, Simona Mocan ${ }^{2}$, Ghenadie Pascarenco ${ }^{3}$, Silvia Drasoveanu ${ }^{1}$, Marinela Galeanu ${ }^{4}$, \\ Daniela Dobru ${ }^{1}$
}

\author{
1) Department of \\ Gastroenterology, Mures \\ County Hospital, University of \\ Medicine and Pharmacy \\ 2) Department of Pathology \\ University of Medicine and \\ Pharmacy \\ 3) Department of Surgery, \\ Mures County Hospital, \\ University of Medicine and \\ Pharmacy, TarguMures \\ 4) Department of \\ Gastroenterology, Municipal \\ Hospital, Campulung \\ Moldovenesc, Suceava \\ Romania
}

\author{
Address for correspondence: \\ Pascarenco Ofelia \\ Mures County Hospital, \\ Gh. Marinescu 1 \\ 540103, TarguMures \\ Romania \\ ofeliapascarenco25@yahoo.com
}

\begin{abstract}
Background \& Aims: Over the past few decades, the incidence of adenocarcinomas of the gastroesophageal junction has rapidly increased. Barrett's esophagus is a risk factor for esophageal adenocarcinoma, but the role of intestinal metaplasia of the gastric cardia as a precursor in cardia-related cancer is controversial. The aims of the present study were to examine the prevalence of intestinal metaplasia in the gastroesophageal junction and to evaluate the clinical, endoscopical and histological features of patients with intestinal metaplasia in the gastric cardia and patients with Barrett's esophagus.

Methods: 286 consecutive patients undergoing gastroduodenoscopy were enrolled in a prospective study. Biopsy specimens were performed in the distal esophagus, squamocolumnar junction, gastric cardia, gastric corpus and antrum.

Results: We identified 44 patients (15.3\%) with intestinal metaplasia in biopsies from gastric cardia and 24 patients $(8.3 \%)$ with Barrett's esophagus. Cardia intestinal metaplasia was significantly associated with older age $(\mathrm{p}=0.03)$, with intestinal metaplasia in the antrum $(\mathrm{p}=0.017)$ and $H$. pylori infection $(\mathrm{p}<0.0001)$. Severe chronic cardia inflammation increased the presence of cardia intestinal metaplasia 6.2 fold $(\mathrm{OR}=6.288$; $\mathrm{p}<0.0001)$. Patients with Barrett's esophagus were predominantly men. Barrett's esophagus presence significantly correlated with reflux symptoms $(\mathrm{p}<0.0001)$, endoscopic esophagitis $(\mathrm{p}<0.0001)$ and hiatal hernia $>2 \mathrm{~cm}(\mathrm{p}=0.002)$. No patient had dysplasia in the gastroesophageal region.

Conclusions: Presence of intestinal metaplasia at the gastroesophageal region correlated with reflux symptoms and endoscopic signs of reflux disease in patients with Barrett's esophagus and with H.pylori infection and distal intestinal metaplasia in patients with cardia intestinal metaplasia.
\end{abstract}

Key words: H. pylori - Barrett's esophagus - cardia intestinal metaplasia - gastric cardia - GERD

Abbreviations: BE- Barrett's esophagus; CIM- cardia intestinal metaplasia; CLE- columnar lined esophagus; GEJ- gastroesophageal junction; GERD - gastroesophageal reflux disease; IM- intestinal metaplasia; LSBE- long segment Barrett's esophagus; SCJ - squamocolumnar junction; SSBE- short segment Barrett's esophagus; USSBE- ultrashort segment Barrett's esophagus.

\section{INTRODUCTION}

Received: 26.11 .2013

Accepted: 26.02.2014
Over the past few decades, while the incidence of distal gastric adenocarcinoma has declined, the incidence of adenocarcinomas of the gastroesophageal junction (GEJ) has increased rapidly in Western countries [1]. These data have raised questions regarding the pathological processes that affect the GEJ. Adenocarcinomas at the gastroesophageal junction appear to arise from foci of incomplete intestinal metaplasia (IM) that develop either in the distal esophagus or in the proximal stomach [2]. Intestinal metaplasia represents the replacement of the normal epithelium of GEJ with intestinal type epithelium as a result of a chronic injury.

There are data to support the viewpoint that chronic inflammation represents the trigger for the development of intestinal metaplasia at the GEJ $[3,4]$. Both gastroesophageal reflux disease (GERD) and $H$. pylori are the major etiologic factors in the development of inflammation and IM of the GEJ [5] and they may coexist in the same patients or may act independently. In GERD patients, IM in the distal esophagus is the result of chronic reflux that causes inflammation of the squamous epithelium, which is replaced by columnar epithelium as a result of a metaplastic reaction, a condition 
known as Barrett's esophagus (BE). The H.pylori infection induces a chronic inflammatory process of the gastric mucosa, followed by atrophic gastritis and the development of IM, which is identified in the distal gastric biopsies, especially at the incisura angularis [6]. The process extends and the chronic inflammation induced by H.pylori involves the gastric mucoasa from the most distal gastric region up to the cardia and the prevalence of IM in the cardia is almost as consistent as the prevalence of IM in the antrum [7,8].

The objectives of the present study were to investigate the prevalence of IM in the GEJ and to evaluate the clinical, endoscopic and histological characteristics of patients with IM in the gastric cardia and of patients with BE.

\section{PATIENTS AND METHODS}

\section{Patients}

Between January 2012 and June 2012, 485 consecutive patients scheduled for upper gastrointestinal (GI) endoscopy in the Gastroenterology Clinic of Tg. Mures County Hospital were invited to participate in this study, irrespective of the indication for the procedure. We excluded from this study patients with previous upper gastrointestinal surgery $(n=42)$, esophageal varices $(n=48)$, coagulation disorders or anticoagulant treatment $(n=22)$, incomplete biopsy protocol - lack of cardiac glandular mucosa in both biopsies of the gastric cardia $(n=57)$. Thirty patients declined to participate or were unable to give informed consent. The remaining 286 patients provided written informed consent and were enrolled in this prospective study with the approval of the Hospital Ethical Committee.

The following information was obtained from every patient: gender, age, history of cigarette smoking, use of alcohol, height, weight. All patients included in this study were asked to report the presence of symptoms indicating gastroesophageal reflux (GE) (heartburn, regurgitation). The reflux symptoms were assessed with a simple yes/no questionnaire whether these had been present twice a week, for a period of at least 3 months.

\section{Endoscopy and biopsy protocol}

The endoscopic examinations were performed by trained endoscopists, who carefully examined the GEJ. The squamocolumnar junction (SCJ) was identified as the demarcation between pearl-grey esophageal squamous epithelium and the red salmon-coloured columnar epithelium while the gastroesophageal junction (GEJ) as the most proximal margin of the gastric folds. Based on the endoscopic appearance of the SCJ, our study population was divided into two groups: first group - a normal appearing GEJ, when the GEJ endoscopically corresponded precisely with the level of the SCJ; the second group - columnar lined esophagus (CLE) when the SCJ was displaced upwards in relation to the GEJ, irrespective of its shape (a straight, regular, circumferential SCJ or irregular with tongue-like protrusion of red mucosa) or its extent.

Endoscopic reflux esophagitis was recorded and graded according to the Los Angeles classification. When the endoscopic exam found a distance of at least $2 \mathrm{~cm}$ between the diaphragmatic hiatus and the gastroesophageal junction, a hiatal hernia was noted.
In our study protocol, biopsy specimens were obtained as follows: 2 biopsies from the esophagus $2 \mathrm{~cm}$ above the SCJ, 2 at the SCJ, 2 from the gastric cardia located within $5 \mathrm{~mm}$ below the SCJ or GEJ in those patients with CLE, 2 biopsies from the gastric corpus, 2 biopsies from the antrum. In patients with CLE, all reddish island-like or tongue-like columnar mucosa in the distal esophagus were biopsied and in the circular CLE segment 4 biopsies were performed of every $\mathrm{cm}$.

\section{Histology}

All biopsy specimens were fixed in formalin and submitted for histopathological examination. All biopsy specimens were then embedded in paraffin, stained with hematoxylineosin and blind-analysed by the same pathologist, for the endoscopic aspect and the biopsy site. The endoscopic biopsies were histologically evaluated regarding the presence of inflammation, $H$. pylori or IM. The presence of goblet cells was the criteria for the diagnosis of IM. Cardiac inflammation (carditis) was classified as either acute (if neutrophils were identified in the lamina propria or glands) or chronic (if the infiltrate of lamina propria was formed by lymphocytes, plasma cells and eosinophils). Based on a visual analogue scale, chronic inflammation of the cardia was graded as mild or severe.

Based both on endoscopic and histologic data, we identified subgroups of patients. Cardiac intestinal metaplasia (CIM) was identified by the presence of goblet cells in gastric cardia specimens, just below the endoscopically normal appearing GEJ. The endoscopic presence of columnar appearing mucosa in the distal esophagus, irrespective of length, with goblet cells on biopsy, was defined as BE and was classified according to its extent in the long segment $\mathrm{BE}$ ( $\mathrm{LSBE})(>3 \mathrm{~cm})$, short segment $\mathrm{BE}$ (SSBE) $(1-3 \mathrm{~cm})$ and ultrashort segment BE (USSBE) $(<1$ $\mathrm{cm})$.

\section{Statistical analysis}

Statistical analysis was performed using the Statistical Package for the Social Sciences, version 20 and Medcalc program version 12.3. Data were classified as nominal variables. For the description of nominal variables frequencies we calculated. For univariate analysis of nominal variables we used $\chi^{2}$. A multivariate analysis was performed using binary logistic regression. Statistical significance was considered when $\mathrm{p}<0.05$.

\section{RESULTS}

A total of 286 patients (Fig. 1) were included in the final analysis with a median age of 59.9 (range 22-87) and a female/ male ratio of 1.1/1. An endoscopically normal appearing GEJ was described in 224 patients $(78.3 \%)$ and CIM was identified in 44 of these patients. Out of the 62 patients (21.6\%) with CLE, 24 had histologic evidence of IM and were classified as patients with BE: 2 patients had LSBE and 22 had SSBE or USSBE. In the $\mathrm{BE}$ group, columnar mucosa had a tongue like protrusion above the GEJ in 14 patients and circular in 10 patients. The patients without histological evidence of IM at GEJ region, both with normal appearing GEJ $(n=180)$ or CLE $(n=38)$, were considered to be the reference group. Men and women were equally distributed in CIM patients, but men were predominant in the BE group:17/24. There were significantly more patients 


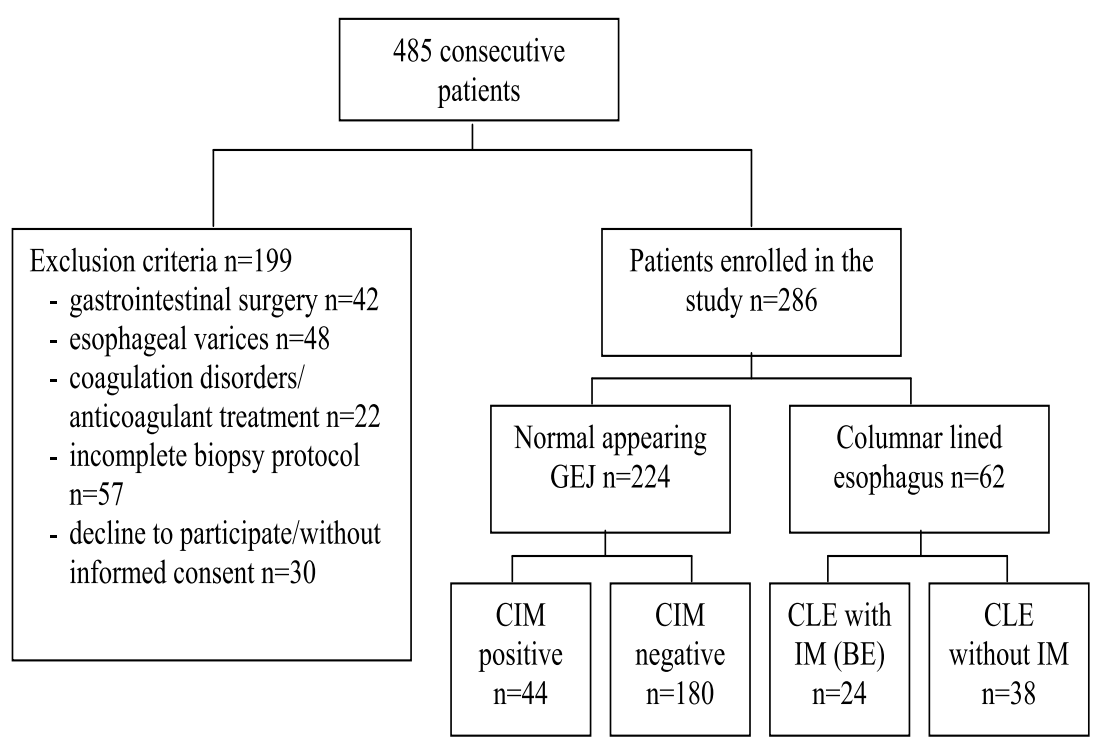

Fig. 1. Flowchart of the population. GEJ - gastroesophageal jonction; CIM - intestinal metaplasia of the cardia; CLE - columnar-lined esophagus; IM - intestinal metaplasia ; BE - Barrett's esophagus

with $\mathrm{BMI}>30 \mathrm{~kg} / \mathrm{m}^{2}$ in the BE group $(\mathrm{p}<0.0001)$ compared with the CIM group: one patient (2.2\%) in the CIM group had $\mathrm{BMI}>30 \mathrm{~kg} / \mathrm{m}^{2}$, compared to 14 out of 24 patients $(58.3 \%)$ in the $\mathrm{BE}$ group; $83.3 \%$ patients from the $\mathrm{BE}$ group $(\mathrm{p}<0.0001)$ were smokers, compared to $27.2 \%$ in the CIM group (Table I).

Out of the 44 patients with CIM, 4 patients (9\%) had GERD symptoms and at endoscopy we only found endoscopic esophagitis in $2(4.5 \%)$ and hiatal hernia in $5(11.3 \%)$ patients. In the $\mathrm{BE}$ group, the majority of patients (19 out of 24 , $79.1 \%)$, had GERD symptoms $(\mathrm{p}<0.0001)$ and the frequency of endoscopic esophagitis and hiatal hernia was $62.5 \%$ $(\mathrm{p}<0.0001)$ and $45.8 \%(\mathrm{p}=0.002)$, respectively, as compared to the CIM patients. Within the reference group, the frequency of endoscopic esophagitis and hiatal hernia was $15.5 \%$ and $27.5 \%$, respectively (Table II).

All 44 patients with CIM had inflammation at the histological analysis of the gastric cardia biopsies $(\mathrm{p}<0.0001)$. Chronic inflammation was present in 43 out of the 44 patients (97.7\%), being classified as severe in 8 patients (18.6\%) and mild in the rest of the 35 patients (81.3\%). Acute inflammation was present in 5 CIM patients, in 4 of them being associated with chronic inflammation. In the BE group, 6 patients (25\%) showed evidence of inflammation in the gastric cardia samples: in 5 cases the inflammation was classified as mild and in one patient as severe. Three patients (12.5\%) in the BE group had also IM in cardia biopsies; all of them had an associated chronic inflammation. Thirty one out of $44(70.4 \%)$ of the patients in the CIM group had IM in the gastric antrum ( $\mathrm{p}=0.017)$, and 10 of these 31 patients had also gastric corpus IM. The BE group and the reference group had similar frequency of antral IM (37.5\% and $36.6 \%$ repectively). H. pylori infection in the distal stomach was significantly more frequent in the CIM group in 26 patients $(59 \%)(\mathrm{p}<0.0001)$, compared to the BE patients: 3 patients in the BE group (12.5\%) and 66 in the reference group (30.2\%). The association of CIM with H.pylori infection in the distal stomach was stronger than that with IM in the distal stomach ( $\mathrm{p}=0.005)$ (Table III).

A complex multivariate analysis showed that H.pylori infection in the distal stomach increased the presence of CIM 4.3 fold $(\mathrm{OR}=4.314 ; \mathrm{p}=0.001)$ and the presence of severe chronic cardia inflammation increased the presence of CIM 6.2 fold $(\mathrm{OR}=6.288 ; \mathrm{p}<0.0001)$.

Table I. Characteristics of the study group

\begin{tabular}{|c|c|c|c|c|c|c|c|}
\hline & & \multirow{2}{*}{$\begin{array}{l}\text { Normal GEJ } \\
\text { with CIM }\end{array}$} & \multicolumn{3}{|c|}{ CLE with IM (BE) } & \multirow{2}{*}{$\begin{array}{l}\text { Reference group (normal } \\
\text { GEJ/CLE without IM) }\end{array}$} & \multirow[t]{2}{*}{$\mathrm{p}^{*}$} \\
\hline & & & LSBE & SSBE & USSBE & & \\
\hline Patients & 286 & $44(15.3 \%)$ & $2(0.69 \%)$ & $17(5.94 \%)$ & $5(1.74 \%)$ & $218(76.2 \%)$ & \\
\hline Age & 59.9 & 60.5 & 46.5 & 67.5 & 52.8 & 59.8 & 0.03 \\
\hline $\mathrm{M} / \mathrm{F}$ & $131 / 155$ & $21 / 23$ & $2 / 0$ & $13 / 4$ & $2 / 3$ & $93 / 125$ & 0.11 \\
\hline $\mathrm{BMI}>30\left(\mathrm{~kg} / \mathrm{m}^{2}\right)$ & 48 & $1(2.2 \%)$ & $1(50 \%)$ & $10(58.8 \%)$ & $3(60 \%)$ & $33(15.1 \%)$ & 0.001 \\
\hline Alcohol & 57 & $8(18.1 \%)$ & $1(50 \%)$ & $3(17.6 \%)$ & $1(20 \%)$ & $44(20.1 \%)$ & 0.68 \\
\hline Cigarette smoker & 122 & $12(27,2 \%)$ & $2(100 \%)$ & $16(94,1 \%)$ & $2(40 \%)$ & $90(41,2 \%)$ & 0.0001 \\
\hline
\end{tabular}

GEJ- gastroesophageal junction; CIM- cardia intestinal metaplasia; CLE- columnar lined esophagus; IM- intestinal metaplasia; BEBarrett's esophagus; LSBE- long segment Barrett's esophagus; SSBE- short segment Barrett's esophagus; USSBE- ultrashort segment Barrett's esophagus. $\mathrm{p}^{*}$ refers to CIM vs CLE with IM patients 
Table II. Clinical and endoscopic characteristics

\begin{tabular}{|c|c|c|c|c|c|c|}
\hline \multirow[t]{2}{*}{ Patients } & \multirow[t]{2}{*}{ CIM } & \multicolumn{3}{|c|}{ CLE with IM (BE) $(n=24)$} & \multirow[t]{2}{*}{ Reference group } & \multirow[t]{2}{*}{$\mathrm{p}^{*}$} \\
\hline & & LSBE & SSBE & USSBE & & \\
\hline $\mathrm{N}$ & 44 & 2 & 17 & 5 & 218 & \\
\hline Symptoms of GERD n(\%) & $4(9 \%)$ & $2(100 \%)$ & $13(76.4 \%)$ & $4(80 \%)$ & $60(27.5 \%)$ & 0.0001 \\
\hline Endoscopic esophagitis (n\%) & $2(4.5 \%)$ & $1(50 \%)$ & $13(76.4 \%)$ & $1(20 \%)$ & $34(15.5 \%)$ & 0.0001 \\
\hline Hiatal hernia (n\%) & $5(11.3 \%)$ & $1(50 \%)$ & $7(41.1 \%)$ & $3(60 \%)$ & $60(27.5 \%)$ & 0.002 \\
\hline
\end{tabular}

GERD - gastroesophageal reflux disease; CIM - intestinal metaplasia of the cardia; CLE - columnar-lined esophagus; IM - intestinal metaplasia; LSBE - long segment Barrett's esophagus; SSBE - short segment Barrett's esophagus; USBE - ultrashort segment Barrett's esophagus. $\mathrm{p}^{*}$ refers to CIM vs CLE with IM patients

Table III. Histological characteristics of patients

\begin{tabular}{lcccccc}
\hline Patients & CIM & \multicolumn{3}{c}{ CLE with IM } & Reference group & $\mathrm{p}^{*}$ \\
\cline { 3 - 5 } & & LSBE & SSBE & USSBE & & \\
\hline $\mathrm{N}$ & 44 & 2 & 17 & 5 & 218 & \\
Carditis & $44(100 \%)$ & 0 & $5(29.4 \%)$ & $1(20 \%)$ & $80(36.6 \%)$ & 0.0001 \\
Antral IM & $31(70.4 \%)$ & 0 & $8(47 \%)$ & $1(20 \%)$ & $85(38.9 \%)$ & 0.017 \\
Antral H.pylori infection & $26(59 \%)$ & 0 & $2(11.7 \%)$ & $1(20 \%)$ & $66(30.2 \%)$ & 0.0001 \\
Corpus IM & $10(22.7 \%)$ & 0 & $1(5.8 \%)$ & 0 & $18(8.25 \%)$ & 0.03 \\
Corpus H.pylori infection & $13(29.5 \%)$ & 0 & $1(5.8 \%)$ & $1(20 \%)$ & $8(3.66 \%)$ & 0.24 \\
Gastric antrum dysplasia & $6(13.6 \%)$ & 0 & 0 & 0 & 0 & 0 \\
Pancreatic metaplasia & 0 & $1(50 \%)$ & $3(17.6 \%)$ & 0 & & 0 \\
\hline
\end{tabular}

Abbreviations: see Table II.

\section{DISCUSSION}

Intestinal metaplasia at the GEJ has incurred much interest in the latter years because of the increased incidence of adenocarcinomas at this site. Barrett's esophagus is a metaplastic abnormality in which normal esophageal squamous mucosa is replaced by columnar epithelium with histological evidence of goblet cells (IM) [9]. The gastric cardia is a subject of controversy among anatomists, histologists, surgeons, oncologists and endoscopists [10], raising an increased interest regarding the etiology (normal congenital structure or acquired condition), pathogenesis and natural history of pathologic conditions involving GEJ. Biopsy specimens from a normalappearing GEJ could also reveal IM within cardiac mucosa, a condition known as CIM [11].

Both BE and CIM have histological evidence of IM with goblet cells, the difference in these conditions being the endoscopically visible tongues of columnar mucosa in the distal esophagus of any length, which would define the lesion as $\mathrm{BE}[12]$.

The prevalence of CIM in the general population at a normal appearing GEJ and its clinical importance is uncertain as the reported data included patients with clinical indications for upper endoscopy. Along several studies, the histological examination of biopsies taken at or below the endoscopically normal GEJ revealed a prevalence of IM varying from 5.9 to $37.3 \%[8,13-15]$. The differences between the reported prevalence rates could relate to several causes: differences in the patients' population studied, in the sites of tissue sampling and biopsy techniques used (antegrade or retrograde position of the endoscope), in the definitions used to describe the endoscopic and anatomic concepts of the GEJ region. In our study, CIM was detected in $15.3 \%$ of the patients, similar to the prevalence reported by El-Zimaity et al [16] (15.4\%) or Wolf et al [17] (15.1\%).

The patients with histological evidence of goblet cells in samples taken when SCJ was displaced upwards in relation to the GEJ, irrespective of its shape or its extent were classified as $\mathrm{BE}$ patients. The prevalence of $\mathrm{BE}$ varies around the world; the data in the reported studies depends on the definition used to describe this condition and on the studied population. Ronkainen et al found BE in $1.6 \%$ of the general Swedish population [18]. Fouad et al [19] found BE in about $7.3 \%$ of patients with chronic GERD symptoms, with a prevalence of $1.2 \%$ for the LSBE and of $6.1 \%$ for the SSBE. In high-risk patients with chronic GERD, Westhoff et al [20] reported a frequency of BE of $13.2 \%$, with an overall frequency of LSBE of $4.8 \%$ and $8.5 \%$ for SSBE. Gerson et al [21] detected BE in $25 \%$ of asymptomatic male veterans older than 50 years of age undergoing screening sigmoidoscopy for CRC, with a prevalence of $7 \%$ in LSBE and $17 \%$ in SSBE. Our study evaluated consecutive, unselected patients undergoing upper endoscopy and found a prevalence of BE of $8.3 \%$. Most of the patients with BE (91.6\%) had SSBE (overall frequency $7.6 \%)$. The overall frequency of LSBE was $0.7 \%$. Taking into consideration that we included unselected patients in our study, the prevalence of $\mathrm{BE}$ could have been higher in a population with chronic reflux symptoms. Previous data [22] reported that patients with $\mathrm{BE}$ were predominantly male. In our study, however, the prevalence of BE did not differ significantly 
between men and women, although males were predominant. Men and women were equally distributed among CIM patients. Patients with CIM were significantly older $(p=0.03)$ than in the $\mathrm{BE}$ group, similar to the data reported by Law et al and Castro et al [14, 23].

Similar to earlier reported findings [24, 25] concerning $\mathrm{BE}$ and GERD association, in our study, patients with BE had significantly higher frequency of GERD symptoms $(\mathrm{p}<0.0001)$, endoscopic esophagitis $(\mathrm{p}<0.0001)$ and hiatal hernia $>2 \mathrm{~cm}$ $(\mathrm{p}=0.002)$, confirming that GERD is an important causal factor which defines a specific group of patients at risk to develop BE. In the recent Pro-Gerd study, the authors reported that the presence of erosive esophagitis, alcohol intake and regular PPI therapy are risk factors, predicting progression towards BE in a population with GERD after 5 years of follow-up[26].

Since some investigators [27] have reported a correlation between CIM and GERD, we analysed this possible association. We found a prevalence of $9 \%$ of GERD symptoms among the patients with CIM, which was lower even compared with the reference group (27.5\%). Furthermore, we found that endoscopic esophagitis and hiatal hernia were more prevalent in the reference group than in the CIM group, suggesting that IM of the gastric cardia is less likely to be a consequence of GERD and that it has a different origin. However, a recent study [28] showed that more than $20 \%$ of GERD patients with IM at a normal GEJ will develop BE within 2-5 years, which could be the link between two entities.

There are abundant data $[14,29,30]$ in the literature revealing an association of CIM with IM in the distal stomach and with H.pylori infection, the difference between these studies being the site of biopsy sampling: biopsies taken at 1-2 $\mathrm{cm}$ below GEJ, below the SCJ, precisely at the GEJ or at the SCJ. In order to obtain more accurate data, we focused only on samples containing cardiac type mucosa. Our findings show a significant correlation between CIM and IM in the antrum $(\mathrm{p}=0.017)$, the association being even stronger between CIM and H.pylori infection in the distal stomach $(\mathrm{p}<0.0001)$. The association of $H$. pylori antrum infection and antral IM had also a significant correlation with CIM $(p=0.005)$. On the other hand, 10 of the 31 patients with CIM and IM in the antrum had concomitant IM in the gastric corpus. In contrast, H. pylori infection in the antrum was found in $12.5 \%$ in $\mathrm{BE}$ patients and in $30.2 \%$ of those in the reference group. Three of our patients $(12.5 \%)$ with BE had also intestinal metaplasia in cardia biopsies.

There is strong evidence to suggest that gastric IM is the response of a chronic injury of the gastric mucosa and the inflammation caused by $H$. pylori infection is the required precursor of IM [31]. An important finding in our CIM patients is that histologic examinations of samples containing cardiac type mucosa revealed inflammation of the cardia (carditis). Carditis was present in all 44 CIM patients (in $97.7 \%$ of these patients it was a chronic inflammation) $(\mathrm{p}<0.0001)$, compared with 6 of 24 patients (25\%) with BE and in $36.6 \%$ of patients in the reference group. In our study, the association of CIM with $H$. pylori infection and carditis is consistent with other studies showing that cardia inflammation is correlated with H. pylori infection, rather than with GERD. Sharma et al [32] discovered data to support $H$. pylori as an etiological agent for carditis, after showing a significant improvement in the degree of inflammation and activity scores in the gastric cardia of patients after $H$. pylori eradication compared to those with persistent infection, without significant changes of IM. Sotoudeh et al [33] found no relation between carditis and reflux esophagitis, but a significant correlation between $H$. pylori and gastritis for all sites of the stomach, stronger for the antrum and cardia inflammation.

The role of CIM as a precursor of cardia-related cancer is still unknown. It seems that CIM is a common finding, maybe even more frequent than estimated, as IM is known to have a patchy distribution. The new endoscopic techniques, such as chromoendoscopy, enhanced magnification endoscopy, narrow band imaging improve mucosal visualization and enable the endoscopists to target the biopsies for a better detection of IM. If in $\mathrm{BE}$ patients the guidelines recommend a close endoscopic surveillance, the management of CIM is unclear: in clinical practice, it is not recommended to take biopsies at or below a normal appearing GEJ. Although we did not find dysplasia in the gastroesophageal region in our patients, there are data to prove an association of dysplasia and IM in GEJ. Sharma et al [34] evaluated the prevalence and incidence rate of dysplasia between patients with SSBE and CIM and found that both were significantly higher in the SSBE group as compared to the CIM group (11.3\% and $4.6 \%$ versus $1.3 \%$ and $1.5 \%$, respectively). Morales et al [35] reported an incidence of CIM dysplasia of $1.4 \%$ /year. In a more recent study [36], on a larger population with a longer follow-up period, no patient with IM-GEJ progressed to esophageal adenocarcinoma, in contrast to $\mathrm{BE}$ patients who had a cumulative risk of progression of $7 \%$ at 10 years. Even if the constant increase of gastric cardia adenocarcinoma has drawn attention to CIM, as shown in these studies, the dysplasia and neoplasia risk in CIM patients is lower than in BE and does not justify an endoscopic surveillance programme for these patients.

A limitation of our study is the fact that the discrimination between IM in the cardia and IM in the SSBE (even $<1 \mathrm{~cm}$ ) was based on the different endoscopic aspects of these entities: SCJ is displaced upwards in relation to the GEJ in patients with BE irrespective of both shape or extent. The use of immunohistochemical stains would have offered a further argument regarding the precise site of the biopsy, since there are studies describing a distinct pattern in cytokeratine 7 and cytokeratine 20 immunostaining in BE patients [37]. We also have to take into account that studies demonstrated a low interobserver reliability coefficients for endoscopic recognition of $\mathrm{BE}<1 \mathrm{~cm}$, suggesting that these segments of Barrett's are difficult to be consistently recognized [38]. Another limitation of the study is the probability of missing BE patients or CIM patients due to sampling errors, as IM has a patchy distribution.

\section{CONCLUSIONS}

This study confirmed that cardia intestinal metaplasia is a common finding, predominantly in older patients and equally prevalent in men and women. Cardia intestinal metaplasia negatively correlated with GERD symptoms, endoscopic esophagitis and hiatal hernia and had a strong association with H.pylori infection, cardia inflammation and distal gastric 
intestinal metaplasia. On the other hand, Barrett's esophagus occurred predominantly in men, and a positive correlation was found with smoking habit, BMI $>30 \mathrm{~kg} / \mathrm{m}^{2}$, GERD symptoms and endoscopic signs of reflux disease.

Conflicts of interest. No conflict to declare.

\section{REFERENCES}

1. Allum WH, Blazeby JM, Griffin SM, et al; Association of Upper Gastrointestinal Surgeons of Great Britain and Ireland, the British Society of Gastroenterology and the British Association of Surgical Oncology. Guidelines for the management of oesophageal and gastric cancer. Gut 2011;60:1449-1472.

2. Spechler SJ. Intestinal metaplasia at the gastroesophageal junction. Gastroenterology 2004;126:567-575

3. Wieczorek TJ, Wang HH, Antonioli DA, Glickman JN, Odze RD. Pathologic features of reflux and Helicobacter pylori-associated carditis: a comparative study. Am J Surg Pathol 2003;27:960-968.

4. Der R, Tsao-Wei DD, Demeester T, et al. Carditis: a manifestation of gastroesophageal reflux disease. Am J Surg Pathol 2001;25:245-252.

5. Odze RD. Unraveling the mystery of the gastroesophageal junction: a pathologist's perspective. Am J Gastroenterol 2005;100:1853-1867.

6. Correa P, Piazuelo MB, Wilson TK. Pathology of gastric intestinal metaplasia: clinical implications. Am J Gastroenterol 2010;105:493-498.

7. Malfertheiner P, Peitz U. The interplay between Helicobacter pylori, gastro-oesophageal reflux disease, and intestinal metaplasia. Gut 2005;54 Suppl 1: i13-i20.

8. Oksanen A, Sipponen P, Karttunen R, Rautelin H. Inflammation and intestinal metaplasia at the squamocolumnar junction in young patients with or without Helicobacter pylori infection. Gut 2003;52:194-198.

9. Phillips WA, Lord RV, Nancarrow DJ, Watson DI, Whiteman DC. Barrett's Esophagus. J Gastroenterol Hepatol 2011;2:639-648.

10. Fregnani JH; Macea JR; Barros MD, et al. Cardia: at the End of the Day, what is it? Int J Morphol 2007;25:375-380.

11. DeMeester SR, DeMeester TR. Columnar mucosa and intestinal metaplasia of the esophagus - fifty years of controversy. Ann Surg 2000;231:303-321.

12. White NM, Gabril M, Ejeckam G, et al. Barrett's esophagus and cardiac intestinal metaplasia: two conditions within the same spectrum. Can J Gastroenterol 2008;22:369-375.

13. Hirota WK, Loughney TM, Lazas DJ, Maydonovitch CL, Rholl V, Wong RK. Specialized intestinal metaplasia, dysplasia, and cancer of the esophagus and esophagogastric junction: prevalence and clinical data. Gastroenterology 1999;116:277-285.

14. Law S, Lam KY, Chu KM, Wong J. Specialized intestinal metaplasia and carditis at the gastroesophageal junction in Chinese patients undergoing endoscopy. Am J Gastroenterol 2002;97:1924-1929.

15. Guelrud M, Herrera I, Essenfel H, Castro J, Antonioli DA. Intestinal metaplasia of the gastric cardia: A prospective study with enhanced magnification endoscopy. Am J Gastroenterol 2002;97:584-589.

16. El-Zimaity HM, Verghese VJ, Ramchatesingh J, Graham DY. The gastric cardia in gastro-oesophageal disease. J Clin Pathol 2000;53:619-625.

17. Wolf C, Seldenrijk CA, Timmer R, Breumelhof R, Smout AJ. Epidemiological, clinical, and histological characteristics of intestinal metaplasia at the oesophagogastric junction compared to short segments of columnar-lined oesophagus. Eur J Gastroenterol Hepatol 2001;13:113-120.
18. Ronkainen J, Aro P, Storskrubb T, et al. Prevalence of Barrett's esophagus in the general population: an endoscopic study. Gastroenterology 2005;129:1825-1831.

19. Fouad YM, Makhlouf MM, Tawfik HM, El-Amin H, Ghany WA, El-Khayat HR. Barrett's esophagus: prevalence and risk factors in patients with chronic GERD in Upper Egypt. World J Gastroenterol 2009;15:3511-3515.

20. Westhoff B, Brotze S, Weston A, et al. The frequency of Barrett's esophagus in high-risk patients with chronic GERD. Gastrointest Endosc 2005;61:226-231.

21. Gerson LB, Shetler K, Triadafilopoulos G. Prevalence of Barrett's esophagus in asymptomatic individuals. Gatroenterology 2002;123:461467.

22. Yachimski P, Lee RA, Tramontano A, Nishioka NS, Hur C. Secular trends in patients diagnosed with Barrett's esophagus. Dig Dis Sci 2010;55:960-966.

23. Castro ML, Fachal C, Pineda JR, et al. Intestinal metaplasia at the esophagogastric junction. Prevelence and association in patients undergoing endoscopy. Gastroenterol Hepatol 2002;25:487-492.

24. Wild C, Hardie LJ. Reflux, Barrett's oesophagus and adenocarcinoma: burning questions. Cancer 2003;3:676-684.

25. Hak NG, Mostafa M, Salah T, et al. Acid and bile reflux in erosive reflux disease, non-erosive reflux disease and Barrett's esophagus. Hepatogastroenterology 2008;55:442-447.

26. Malfertheiner P, Nocon M, Vieth M, et al. Evolution of gastrooesophageal reflux disease over 5 years under routine merdical care-- the ProGERD study. Aliment Pharmacol Ther 2012;35:154-164.

27. Carton E, Caldwell MT, McDonald G, Rama D, Tanner WA, Reynolds JV. Specialized intestinal metaplasia in patients with gastro-oesophageal reflux disease. Br J Surg 2000;87:116-121.

28. Leodolter A, Nocon M, Vieth M, et al. Progression of specialized intestinal metaplasia at the cardia to macroscopically evident Barrett's esophagus: an entity of concern in the ProGERD study. Scand J Gastroenterol 2012;47:1429-1435.

29. Morini S, Zullo A, Hassan C, Lorenzetti R, Stella F, Martini MT. Gastric cardia inflammation: role of Helicobacter pylori infection and symptoms of gastroesophageal reflux disease. Am J Gastroenterol 2001;96:2337-2340.

30. Pieramico, O, Zanetti, MV. Relationship between intestinal metaplasia of the gastro-oesophageal junction, Helicobacter pylori infection and gastro-oesophageal reflux disease: a prospective study. Dig Liver Dis 2000;32:567-572.

31. Tulassay Z, Stolte M, Engstrand L, et al. Twelve-month endoscopic and histological analysis following proton-pump inhibitor-based triple therapy in Helicobacter pylori-positive patients with gastric ulcers. Scand J Gastroenterol 2010;45:1048-1058.

32. Sharma P, Topalovski M, Mayo MS, Sampliner RE, Weston AP. Helicobacter pylori eradication dramatically improves inflammation in the gastric cardia. Am J Gastroenterol 2000;95:3107-3111.

33. Sotoudeh M, Derakhshan MH, Abedi-Ardakani B, et al. Critical role of Helicobacter pylori in the pattern of gastritis and carditis in residents of an area with high prevalence of gastric cardia cancer. Dig Dis Sci 2008;53:27-33.

34. Sharma P, Weston AP, Morales T, Topalovski M, Mayo MS, Sampliner RE. Relative risk of dysplasia for patients with intestinal metaplasia in the distal oesophagus and in the gastric cardia. Gut 2000;46:9-13.

35. Morales TG, Camargo E, Bhattacharyya A, Sampliner RE. Longterm follow-up of intestinal metaplasia of the gastric cardia. Am J Gastroenterol 2000;95:1677-1680. 
36. Jung KW, Talley JN, Romero Y, et al. Epidemiology and natura history of intestinal metaplasia of the gastroesophageal junction and Barrett's esophagus: a population-based study. Am J Gastroenterol 2011;106:1447-1455.

37. Sarbia M, Donner A, Franke C, Gabbert HE. Distinction between intestinal metaplasia in the cardia and in Barrett's esophagus: the role of histology and immunohitochemistry. Hum Pathol 2004;35:371376.

38. Sharma P, Dent J, Armstrong D, et al. The development and validation of an endoscopic grading system for Barrett's esophagus: the Prague C \& M criteria. Gastroenterology 2006;131:1392-1399. 